MSM are residents of the district and are above the age 18 years at the time the study was conducted with voluntary willingness to participate in the study without any financial compensation. The collected data were compared and analyzed through regression-model using health-belief-model.

Results More than $75 \%$ of respondents reported that cultural belief is used as a tool that prevented them from accessing health-care-services, impacted on accessing prevention and treatment.

$60 \%$ of clinicians and $80 \%$ of non-clinicians had insufficient knowledge about MSM, using culture to promoted stigma/discrimination. $60 \%$ of the respondent experienced physical/verbal abuse' in their community fueled by cultural beliefs. Culture is used as a yardstick to manhood, denying MSM of their cultural rites.

Conclusions Cultural influence on MSM health has shown to be very vast affecting the perceptions of their overarching health mostly impacting on their mental health. Health inequality is linked to direct access to health services and the provision of quality health services for MSM hindered by cultural beliefs is used as a deterrent yardstick. Community role players engagement and support identified as a tool to reduce cultural misconception against MSM

\section{P438 INCIDENCE AND OUTCOMES OF JARISCH-HERXHEIMER REACTIONS FOLLOWING TREATMENT FOR INFECTIOUS SYPHILIS IN LATE PREGNANCY IN ALBERTA, CANADA, 2015-2020}

${ }^{1} S$ Macumber*, ${ }^{1} \mathrm{~A}$ Singh, ${ }^{1} \mathrm{~J}$ Robinson, ${ }^{2} \mathrm{P}$ Smyczek, ${ }^{1} \mathrm{C}$ Sklar, ${ }^{2} \mathrm{~J}$ Gratrix, ${ }^{2} \mathrm{~L}$ Rathjen. ${ }^{1}$ University of Alberta, Edmonton, Canada; ${ }^{2}$ Alberta Health Services, Edmonton, Canada

10.1136/sextrans-2021-sti.455

Background In Alberta (Canada), infectious syphilis rose from 4:100,000 in 2014 to $52: 100,000$ in 2019 with a parallel rise in congenital syphilis. Treatment of pregnant women is highly efficacious but can precipitate the Jarisch-Herxheimer (JH) reaction. Symptoms of $\mathrm{JH}$ reaction are often transient but may include fever, uterine contractions, fetal heart rate abnormalities, and preterm labor. Because of these observations, many guidelines recommend hospitalization for fetal monitoring be considered. We sought to determine the incidence, and severity of $\mathrm{JH}$ reactions and the maternal and fetal outcomes following treatment.

Methods Retrospective cohort study of pregnant women at $>20$ weeks gestation admitted to urban referral centers for treatment of infectious syphilis between 2015 and 2020. Data was extracted from a provincial database and supplemented by a review of medical records. Data collection was completed in March 2021.

Results 39 women received benzathine penicillin $G$ for the treatment of infectious syphilis in pregnancy. One mild $\mathrm{JH}$ reaction was identified in a patient with early latent syphilis. There were no serious maternal or fetal outcomes attributable to treatment. Average gestational age at treatment and delivery was 26 and 37 weeks respectively. Ten cases were staged as primary, 4 as secondary, and 25 as early latent syphilis. Five cases of confirmed congenital syphilis were identified. Further analysis of results is underway.
Conclusions Data from our study challenges the recommendation for routine admission for the treatment of infectious syphilis in late pregnancy as no moderate or severe $\mathrm{JH}$ reactions were identified in our cohort. Further research is required to determine if a subgroup of women not identified in our cohort are at risk for moderate or severe $\mathrm{JH}$ reaction. These findings are timely given the global resurgence of infectious syphilis as inpatient resources continue to be strained by the ongoing COVID-19 pandemic.

\section{P440 COVID-19 ANXIETY AND SEXUAL BEHAVIOUR AMONG MALE AND GENDER-DIVERSE PEOPLE WHO HAVE SEX WITH MEN (MGDPSM) DURING ENGLAND'S FIRST LOCKDOWN}

${ }^{1} \mathrm{~N}$ Edelman*, ${ }^{2,3} \mathrm{~W}$ Nutland, ${ }^{4} \mathrm{~T}$ Witzel, ${ }^{3} \mathrm{P}$ Samba, ${ }^{5} \mathrm{~T}$ Nadarzynski. ${ }^{1}$ University of Brighton, Brighton and Hove, UK; ${ }^{2}$ London School of Hygiene and Tropical Medicine, London, UK; ${ }^{3}$ The Love Tank CIC, London, UK; ${ }^{4}$ Department of Public Health, Environments and Society, London School of Hygiene and Tropical Medicine, London, UK; ${ }^{5}$ School of Social Sciences, University of Westminster, London, UK

\subsection{6/sextrans-2021-sti.456}

Background In response to the COVID-19 pandemic, a lockdown was imposed in England March-June 2020, requiring people to cease outside physical contact except for essential reasons. A study was conducted to understand the impact on mental and sexual wellbeing among Male and Gender-Diverse People who have Sex with Men.

Methods A structured anonymous survey was conducted online, advertised via social media using a convenience sampling approach. Participants were asked about use of sexual health interventions and sexual networking apps, sexual behaviour and COVID-19. Qualitative interview participants were purposively sampled from survey participants who provided contact details for that purpose.

Results $\mathrm{N}=1429$ MGDPSM completed the survey (MarchJuly 2020). $N=15$ survey respondents who reported having casual sex partners during lockdown participated in an interview (August 2020-February 2021). $\mathrm{N}=331$ (26.5\%) reported feeling anxious about COVID-19 more than half of the time. $\mathrm{N}=155(12.1 \%)$ reported 1 casual sex partner during lockdown, and $\mathrm{n}=156 \quad(12.2 \%)$ reported $2+$. $\mathrm{N}=1014$ (77.6\%) reported decreasing their number of casual sex partners during lockdown. This was not associated with COVID-19 anxiety (Chi-square $=0.82 \mathrm{p}=0.36$ ) or belief about being infected (Chi-square $=1.77 \mathrm{p}=0.41$ ). Qualitative findings suggest desire to comply with guidance was a key motivator in reducing casual partner numbers, and that this was facilitated by using COVID-19 risk-reduction strategies such as having virtual sex.

Conclusion The majority of survey respondents reduced casual partner numbers. This may be explained by desire to adhere to physical distancing measures which was not measured but which emerged as a key motivator among interviewees. As the pandemic continues alongside growing lockdown fatigue, future research is needed to identify and monitor motivations to reduce casual partner numbers during future physical distancing requirements, and to ensure health promotion highlights self-protective strategies available to MGDPSM. 